MATEC Web of Conferences 41, 05003 (2016)

DOI: $10.1051 /$ matecconf/20164105003

(C) Owned by the authors, published by EDP Sciences, 2016

\title{
On path hypercompositions in graphs and automata
}

\author{
Christos G. Massouros ${ }^{a}$ \\ Technological Institute of Sterea Hellas, Evia GR 34400, Greece
}

\begin{abstract}
The paths in graphs define hypercompositions in the set of their vertices and therefore it is feasible to associate hypercompositional structures to each graph. Similarly, the strings of letters from their alphabet, define hypercompositions in the automata, which in turn define the associated hypergroups to the automata. The study of the associated hypercompositional structures gives results in both, graphs and automata theory.
\end{abstract}

\section{Introduction}

An operation or composition in a non-void set $H$ is a function from $H \times H$ to $H$ while a hyperoperation or hypercomposition is a function from $H \times H$ to the power set $\mathscr{P}(H)$ of $H$. An algebraic structure that satisfies the axioms:

$$
\begin{array}{lll}
\text { i. } a(b c)=(a b) c & \text { for every } a, b, c \in H & \text { (associativity) } \\
\text { ii. } a H=H a=H & \text { for every } a \in H & \text { (reproductivity) }
\end{array}
$$

is called group if $« \cdot »$ is a composition, and hypergroup [6] if $« \cdot »$ is a hypercomposition [13]. A set $H$ endowed with a hypercomposition "." is called hypergroupoid if $x y \neq \varnothing$ for all $x, y$ in $H$, otherwise it is called partial hypergroupoid. If $A$ and $B$ are non-empty subsets of $H$, then $A B$ signifies the union $\bigcup_{(a, b) \in A \times B} a b$. If $A=\varnothing$ or $B=\varnothing$, then $A B=\varnothing . \quad A b$ and $a B$ will have the same meaning as $A\{b\}$ and $\{a\} B$. A hypergroupoid is called semihypergroup if only (i) is valid, while it is called quasihypergroup if only (ii) holds. A hypercomposition is called closed (or containing) if the two participating elements are included in the result. A hypercomposition is called right closed if $a \in b a$ for all $a, b \in H$ and left closed if $a \in a b$ for all $a, b \in H$. A hypercomposition is called right open if $a \notin b a$ for all $a, b \in H$ with $b \neq a$. The definition of left open hypercomposition is similar. Obviously, a hypercomposition is open if it is both right and left open. In [13] the following propositions has been proved:

Proposition 1. The hypercomposition in a hypergroup $H$ is right closed if and only if $a / a=H$ for all $a \in H$, while it is left closed if and only if $a \backslash a=H$ for all $a \in H$.

Proposition 2. The hypercomposition in a hypergroup $H$ is right open if and only if $a / a=a$ for all $a \in H$, while it is left open if and only if $a \backslash a=a$ for all $a \in H$.
Proposition 3. If the hypercomposition in a hypergroup $H$ is right or left open, then all its elements are idempotent .

Two induced hypercompositions (the left and the right division) derive from the hypercomposition of the hypergroup [6], i.e.

$$
a / b=\{x \in H \mid a \in x b\} \text { and } b \mid a=\{y \in H \mid a \in b y\}
$$

When "." is commutative, $a / b=b \mid a$. Consequences of the axioms (i) and (ii) are:

i. $a b \neq \varnothing$, for all $a, b \in H$.

ii. $a / b \neq \varnothing$ and $a \mid b \neq \varnothing$, for all $a, b \in H$.

iii. the nonempty result of the induced hypercompositions is equivalent to the reproductive axiom.

iv. $(a / b) / c=a /(c \cdot b), c|(b \mid a)=(b \cdot c)| a,(b \mid a) / c=b \mid(a / c)$, for all $a, b, c \in H$ (mixed associativity) [7]

A transposition hypergroupoid is a hypergroupoid which satisfies the axiom [5]:

$$
b \backslash a \cap c / d \neq \varnothing \text { implies } a d \cap b c \neq \varnothing
$$

A commutative transposition hypergroup is called join hypergroup or join space $[5,8]$.

In general graph is a set of points called vertices connected by lines called edges. A path in a graph is a sequence of no repeated vertices $v_{1}, v_{2}, \ldots, v_{n}$, such that $\overline{\mathrm{v}_{1} \mathrm{v}_{2}}, \overline{\mathrm{v}_{2} \mathrm{v}_{3}}, \ldots, \overline{\mathrm{v}_{\mathrm{n}-1} \mathrm{v}_{\mathrm{n}}}$, are edges in the graph. The length of a path is the number of edges that it uses. A graph is said to be connected if every pair of its vertices is connected by a path. A directed graph (or digraph) is a graph, where the edges have a direction associated with them. A degenerate edge of a graph which joins a vertex to itself, also called a self-loop or loop. Multiple edges are two or more edges that connect the same two vertices. The term multigraph refers to a graph which has multiple edges between nodes. A directed graph (or digraph) is a graph, where the edges have a direction associated with them. A simple graph (or strict graph), is an unweighted, undirected graph containing no graph loops or multiple edges. A tree $\mathscr{J}$ is a simple, connected graph with no

\footnotetext{
${ }^{\mathrm{a}}$ Corresponding author: Ch.Massouros@gmail.com
}

This is an Open Access article distributed under the terms of the Creative Commons Attribution License 4.0, which permits unrestricted use distribution, and reproduction in any medium, provided the original work is properly cited. 
cycles. A spanning tree of a connected graph is a tree whose vertex set is the same as the vertex set of the graph, and whose edge set is a subset of the edge set of the graph.

An automaton $\mathscr{t}$ is a collection of five objects $\left(\Sigma, \mathrm{S}, \delta, \mathrm{s}_{0}, \mathrm{~F}\right)$ where $\Sigma$ is the alphabet of input letters (a finite nonempty set of symbols), $\mathrm{S}$ is a finite nonvoid set of states, $\mathrm{s}_{\mathrm{o}}$ is the start (or initial) state, an element of $\mathrm{S}$, $\mathrm{F}$ is the set of the final (or accepting) states, a (possibly empty) subset of $\mathrm{S}$ and $\delta$ is the state transition function with domain $\mathrm{S} \times \Sigma$ and range $\mathrm{S}$, in the case of a deterministic automaton (DFA), or $\mathscr{P}(\mathrm{S})$, in the case of a nondeterministic automaton (NDFA). $\Sigma^{*}$ denotes the set of words (or strings) formed by the letters of $\Sigma$-closure of $\Sigma$ - and $\lambda \in \Sigma^{*}$ signifies the empty word. $\Sigma^{*}$ under the concatenation of words is a monoid, with neutral element $\lambda$, since $\lambda x=x \lambda=x$ for all $x$ in $\Sigma^{*}$. Moreover $\Sigma^{*}$ becomes a hyperingoid under the b-hyperoperation: $x+y=\{x, y\}$ for all $x, y$ in $\Sigma^{*}$ [20]. Given a DFA $\mathscr{\mathscr { t }}$, the extended state transition function for $\mathscr{t}$, denoted $\delta^{*}$, is a function with domain $\mathrm{S} \times \Sigma^{*}$ and range $\mathrm{S}$ defined recursively as follows:

i. $\delta^{*}(s, a)=\delta(s, a)$ for all $s$ in $\mathrm{S}$ and $a$ in $\Sigma$

ii. $\delta^{*}(s, \lambda)=s$ for all $s$ in $\mathrm{S}$

iii. $\delta^{*}(s, a x)=\delta^{*}(\delta(s, a), x)$ for all $s$ in $\mathrm{S}, x$ in $\Sigma^{*}$ and $a$ in $\Sigma$.

P. Corsini [2], M. Gionfriddo [4], Nieminen [21, 22], I. Rosenberg [23], M. De Salvo et al. [24] and others studied hypergroups associated with graphs. G. G. Massouros [14-18] and after him J. Chvalina [1] studied hypergroups associated with automata. Moreover, in [15] G. G. Massouros introduced the path hypercomposition in graphs and subsequently Ch. G. Massouros and G. G. Massouros introduced in [9] another type of path hypercomposition in graphs and some relevant hypercompositions in automata.

\section{The path hypercompositions in Graphs}

In the set $V$ of the vertices of a tree, a hypercompostion "." has been introduced in [9] as follows: for each two vertices $x, y$ in $V, x \cdot x=x$ and $x \cdot y$ is the set of all vertices which belong to the path that connects vertex $x$ with vertex $y$. Since tree is an undirected graph, this hypercomposition is commutative. Furthermore, this hypercomposition is a closed hypercomposition. Therefore:

Proposition 4. If $V$ is the set of the vertices of a tree $\mathscr{T}$, then $V=x / x$, for each $x$ in $V$.

The set $\langle x, y\rangle=x / y \cup x \cdot y \cup y / x$, where $x \neq y$ are two vertices of $\mathscr{T}$, is called the line of $\mathscr{J}$ which is defined by $x, y$. A subset $S$ of $V$ is called convex, if it holds $x \cdot y \subseteq S$, for each $x, y$ in $S$. In [9] it is proved that the lines of $\mathscr{J}$ are convex sets. Moreover the following important theorem it is proved in [9]:

Theorem 1. If $V$ is the set of the vertices of a tree $\mathscr{T}$, then $(V$, ) is a join space.

It is known that any connected graph has at least one spanning tree and that there exist algorithms which find such trees. Hence any graph can be endowed with the join space structure through its spanning trees.

Theorem 2. Let G be a connected graph and $\mathcal{T} a$ spanning tree of $G$. The set of the vertices of the graph becomes a join space if for all vertices $x, y$ of $\mathcal{G}$, the hypercomposition $x \cdot y$ is the set of all vertices which belong to the path that connects vertex $x$ with vertex $y$ in T.

Since a graph may have more than one spanning trees, more than one join spaces can be associated to a graph.

Next, define in the set $V$ of the vertices of a tree $\mathscr{J}$ a hypercomposition "•", such that for each two vertices $x, y$ in $V, x \bullet y$ consists of all the internal vertices which belong to the path that connects vertex $x$ with vertex $y$, that is, if $\overline{x v_{1}}, \overline{v_{1} v_{2}}, \ldots, \overline{v_{n} y}$ are edges in a path connecting the vertices $x$ and $y$, then $x \cdot y=\left\{v_{1}, v_{2}, \ldots, \mathrm{v}_{n}\right\}$. This hypercomposition is an open hypercomposition. It is obvious that $(V, \bullet)$ is a partial hypergoupoid, since the result of the hypercomposition of two successive vertices is void. If the above hypercomposition is introduced in a simple connected graph, then it is possible to exist more than one paths connecting two vertices $x, y$ of the graph. Hence if $\overline{x v_{1}}, \overline{v_{1} v_{2}}, \ldots, \overline{v_{n} y}$ are edges in a path which connects the vertices $x$ and $y$, then $\left\{v_{1}, v_{2}, \ldots, \mathrm{v}_{n}\right\} \subseteq x \bullet y .(V, \bullet)$ will be a hypergroupoid if and only if for any two vertices $x$ and $y$ of $V$ there exists a path from $x$ to $y$ of length greater or equal to 2 .

The Boolean domain $\mathrm{B}=\{0,1\}$ becomes a semiring under the addition

$$
0+1=1+0=1+1=1,0+0=0
$$

and the multiplication

$$
0 \cdot 0=0 \cdot 1=1 \cdot 0=0, \quad 1 \cdot 1=1 .
$$

This semiring is called a binary Boolean semiring. A Boolean matrix is a matrix with entries from the binary Boolean semiring. A square Boolean matrix is called total if all its entries are equal to 1 [10]. The adjacency matrix of a graph on $n$ vertices is an $n \times n$ Boolean matrix $A=\left(a_{i, j}\right)$ in which the entry $a_{i, j}$ equals to 1 , if there is an edge from vertex $i$ to vertex $j$ and equals to 0 if there is no edge from vertex $i$ to vertex $j$. Through the adjacency matrix a binary relation $\rho$ can be defined in in the set $V$ of the vertices as follows:

$$
\left(a_{i}, a_{j}\right) \in \rho \text { if and only if } a_{i j}=1
$$

In [11] the following theorem has been proved:

Theorem 3. Let $H$ be a non-empty set and $\rho$ a binary relation on $H$. Then Corsini's hypercomposition in $H$ :

$$
x y=\{z \in H \mid(x, z) \in \rho \text { and }(z, y) \in \rho\}
$$

endows $H$ with the hypergroup structure if and only if $(x, y) \in \rho$, for all $x, y \in H$.

A consequence of the above theorem is the following theorem:

Theorem 4. Let $V$ be the set of the vertices of a graph $\mathcal{G}$. Then the hypercomposition in $V$ : 


$$
x \cdot y=\left\{\begin{array}{l}
\{z \in H \mid x, z, y \text { is a path in } \mathscr{Z}\} \text { if } x \neq y \\
x \text { if } x=y
\end{array}\right.
$$

endows $V$ with the hypergroup structure if and only if the adjacency matrix of G is total.

\section{The path hypercompositions in Automata}

In [14-19] it has been shown by G. Massouros, that the set of the states of an automaton, equipped with different hypercompositions, can be endowed with the structure of the hypergroup. The hypergroups that have derived in this way were named attached hypergroups to the automaton. Up to this point several kinds of attached hypergroups have introduced in order to describe the structure and the operation of the automata with the use of tools from the Hypercompositional Algebra. Among them there are:

i. the attached hypergroups of the order, and

ii. the attached hypergroups of the grade.

These two kinds of hypergroups have also been used for the minimization of the automata.

Moreover, in [15] another hypergroup, which derived through a different consideration of the hypercomposition, has been attached to the set of the states of an automaton. Due to its definition this hypergroup was named by G. Massouros attached hypergroup of the paths and it has led to a new proof of Kleene' s theorem. Furthermore, in [16], the attached hypergroup of the operation has been attached to the automaton. Apart from the other results, this hypergroup can indicated all the states in which an automaton can be found after the $t$ clock pulse.

Hereafter two hypercompositions will be presented which are defined through the strings of letters from the alphabet of the automaton. Let $\mathscr{\mathscr { t }}$ be the automaton $\left(\Sigma, \mathrm{S}, \delta, \mathrm{s}_{0}, \mathrm{~F}\right)$. If $x$ be a word in $\Sigma^{*}$, then:

$\operatorname{Prefix}(x)=\left\{y \in \Sigma^{*} \mid y z=x\right.$ for some $\left.z \in \Sigma^{*}\right\}$

and $\quad \operatorname{Suffix}(x)=\left\{z \in \Sigma^{*} \mid y z=x\right.$ for some $\left.y \in \Sigma^{*}\right\}$

Let $\mathrm{s}$ be an element of $\mathrm{S}$. Then

$$
\mathrm{I}_{\mathrm{s}}=\left\{x \in \Sigma^{*} \mid \delta^{*}\left(\mathrm{~s}_{\mathrm{o}}, x\right)=\mathrm{s}\right\}
$$

and $\quad \mathrm{P}_{\mathrm{s}}=\left\{\mathrm{s}_{\mathrm{i}} \in \mathrm{S} \mid \mathrm{s}_{\mathrm{i}}=\delta^{*}\left(\mathrm{~s}_{0}, y\right), y \in \operatorname{Prefix}(x), x \in \mathrm{I}_{\mathrm{s}}\right\}$

Considering the automaton as a directed graph, $\mathrm{P}_{\mathrm{s}}$ is the set of the states which appear in all possible paths connecting the start state $\mathrm{s}_{\mathrm{o}}$ with the state s. Since $\lambda \in \Sigma^{*}$ the states $\mathrm{s}_{\mathrm{o}}$ and $\mathrm{s}$ are in $\mathrm{P}_{\mathrm{s}}$. In the set of the states of $\mathscr{\mathscr { C }}$ we introduce the hypercomposition

$$
\mathrm{s}+\mathrm{q}=\mathrm{P}_{\mathrm{s}} \cup \mathrm{P}_{\mathrm{q}} \text { for all } \mathrm{s}, \mathrm{q} \in \mathrm{S}
$$

This hypercomposition is commutative, thus the two induced hypercompositions coincide and so we have:

$$
\mathrm{s} / \mathrm{q}=\mathrm{q} \backslash \mathrm{s}=\left\{\begin{array}{l}
\mathrm{S}, \text { if } \mathrm{s} \in \mathrm{P}_{\mathrm{q}} \\
\left\{\mathrm{r} \in \mathrm{S} \mid \mathrm{P}_{\mathrm{s}} \subseteq \mathrm{P}_{r}\right\} \text { if } \mathrm{s} \notin \mathrm{P}_{\mathrm{q}}
\end{array}\right.
$$

It is proved that [9]:

Proposition 5. The set $S$ endowed with the hypercomposition (1) is a join hypergroup.

The other hypercomposition is defined as follows:

$$
\mathrm{s}+\mathrm{q}=\mathrm{P}_{\mathrm{s}} \cap \mathrm{P}_{\mathrm{q}} \text { for all } \mathrm{s}, \mathrm{q} \in \mathrm{S}
$$

Since $s_{0} \in P_{r}$ for all $r \in S$, the results of hypercomposition (2) are always non void sets. Moreover this hypercomposition is commutative, thus the two induced hypercompositions coincide and so we have:

$$
\mathrm{s} / \mathrm{q}=\mathrm{q} \backslash \mathrm{s}= \begin{cases}\mathrm{S}, & \text { if } \mathrm{s} \in \mathrm{P}_{\mathrm{q}} \\ \varnothing, & \text { if } \mathrm{s} \notin \mathrm{P}_{\mathrm{q}}\end{cases}
$$

It has been proved that [9]:

Proposition 6. The set $S$ endowed with the hypercomposition (2) is a join semihypergroup.

Acknowledgments. This research has been co-financed by the European Union (European Social Fund - ESF) and Greek national funds through the Operational Program "Education and Lifelong Learning" of the National Strategic Reference Framework (NSRF) Research Funding Program: ARCHIMEDES III. Investing in knowledge society through the European Social Fund.

\section{References}

1. J. Chvalina, L. Chvalinova, State hypergroups of Automata, Acta Mathematica et Informatica Univ. Ostraviensis 4, pp. 105-120 (1996).

2. P. Corsini, Graphs and Join Spaces, J. of Combinatorics, Information and System Sciences, 16, 4, pp. 313-318 (1991).

3. P. Corsini, Binary relations and hypergroupoids, Italian J. Pure \& Appl. Math., 7, pp. 11-18 (2000).

4. M. Gionfriddo, Hypergroups associated with multihomomorphisms between generalized graphs, Convegno su sistemi binary e loro applicazioni, edit. P. Corsini, Taormina 1978, pp. 161-174 (1978).

5. J. Jantosciak, Transposition hypergroups, Noncommutative Join Spaces, Journal of Algebra, 187, pp. 97119 (1997).

6. F. Marty, Sur in generalisation de la notion de group, Huitieme Congres des Matimaticiens scad, Stockholm 1934, pp. 45-59 (1934).

7. Ch.G. Massouros, On the semi-subhypergroups of a hypergroup, Internat. J. Math. \& Math. Sci. 14, 2, pp. 293-304 (1991).

8. Ch.G. Massouros, Canonical and Join Hypergroups, An. Stiintifice Univ. "Al. I. Cuza", Iasi, Tom. XLII, Matematica, fasc.1, pp. 175-186 (1996).

9. Ch.G. Massouros, G.G. Massouros, Hypergroups Associated with Graphs and Automata, ICNAAM 2009, AIP Conference Proceedings, pp. 164-167.

10. Ch.G. Massouros, Ch. Tsitouras, S. Voliotis, Square Roots of Total Boolean Matrices - Enumeration issues, ICSSIP 2009, IEEE Conference Publishing doi:10.1109/IWSSIP.2009.5367718

11. Ch.G. Massouros, Ch. Tsitouras, Enumeration of hypercompositional structures defined by binary relations, Italian Journal of Pure and Applied Mathematics, 28, pp. 43-54 (2011).

12. Ch. Tsitouras, Ch.G. Massouros, Enumeration of Rosenberg type Hypercompositional structures defined by binary relations, European Journal of Combinatorics, 33, 1, pp. 1777-1786, (2012).

13. Ch.G. Massouros, On connections between vector spaces and hypercompositional structures Italian 
Journal of Pure and Applied Mathematics, 34, pp. 133-150, (2015)

14. G.G. Massouros, J. Mittas, Languages - Automata and hypercompositional structure, Proceedings of the $4^{\text {th }}$ Internat. Cong. on Algebraic Hyperstructures and Applications, World Scientific, pp. 137-147 (1991).

15. G.G. Massouros, Automata and Hypermoduloids, Proceedings of the $5^{\text {th }}$ Inter. Cong. in Algebraic Hyperstructures and Applications, Hadronic Press, pp. 251-266 (1994).

16. G.G. Massouros, An Automaton during its operation, Proceedings of the $5^{\text {th }}$ Inter. Cong. in Algebraic Hyperstructures and Applications, Hadronic Press, pp. 267-276 (1994).

17. G.G. Massouros, Hypercompositional Structures in the Theory of the Languages and Automat, An. stiintifice Univ. Al. I. Cuza, Iasi, Informatica, t. iii, pp. 65-73 (1994).

18. G.G. Massouros, Hypercompositional Structures from the Computer Theory, Ratio Matematica, 13, pp. 37-42, (1999).

19. G.G. Massouros, On the attached hypergroups of the order of an automaton, Journal of Discrete Mathematical Sciences \& Cryptography, 6, no 2-3, pp. 207-215, (2003).

20. G.G. Massouros, The Hyperringoid, Multiple Valued Logic, 3, pp. 217-234 (1998).

21. J. Nieminen, Join Space Graph, Journal of Geometry, 33, pp. 99-103 (1988).

22. J. Nieminen, Chordal Graphs and Join Spaces, Journal of Geometry, 34, pp. 146-1511989,.

23. I. Rosenberg, Hypergroups induced by paths of a directed graph, Italian J. of Pure and Appl. Math. 4, pp. 133-142 (1998).

24. M. De Salvo, D. Fasino, D. Freni, G. Lo Faro, Fully simple semihypergroups, transitive digraphs, and sequence A000712, Journal of Algebra, 415, pp. 6587 (2014). 